\title{
四姑娘山国家级自然保护区鸟兽多样性初步调查
}

\author{
胡茜茜 ${ }^{1}$ 郑维超 ${ }^{2}$ 李佳琦 $^{3}$ 李 暏 ${ }^{4}$ 杨 晗 ${ }^{5}$ 陈 星 ${ }^{1}$ 官天培 $^{*}$ \\ 1 (绵阳师范学院生态安全与保护四川省重点实验室, 四川绵阳 621000) \\ 2 (唐家河国家级自然保护区, 四川广元 628100) 3 (生态环境部南京环境科学研究所, 南京 210042) \\ 4 (北京大学生命科学学院, 北京 100871) 5 (四姑娘山国家级自然保护区, 四川阿坝 624200)
}

\begin{abstract}
摘要: 物种的分布与多度对我们理解生态过程、实施保护管理以及评估全球变化的影响都非常重要。2017年5-12 月, 我们按照公里网格在四川省四姑娘山国家级自然保护区共布设60台固定位点红外相机, 调查了保护区内主要 沟系的兽类和鸟类多样性。本次调查累计 11,013 个相机工作日, 共拍摄到31种兽类和鸟类, 隶属7目18科, 包括5种 国家一级和10种国家二级重点保护野生动物, 被IUCN红色名录评估为濒危( EN)、易 (VU)和近危(NT)的野生动物 分别有2种、 5 种和 3 种。其中, 相对多度指数居于兽类前五位的是毛冠鹿(Elaphodus cephalophus)、水鹿(Rusa unicolor)、喜马拉雅旱獭(Marmota himalayana)、野猪 (Sus scrofa)、猪獾(Arctonyx collaris); 相对多度指数居于鸟类 前五位的则是血雉(Ithaginis cruentus)、白马鸡(Crossoptilon crossoptilon)、大噪鹊(Garrulax maximus)、雉鹑 (Tetraophasis obscurus)和灰头鸫(Turdus rubrocanus)。由于局域性气候、植被类型和地形结构的不同，使四姑娘山 国家级自然保护区与毗邻的卧龙国家级自然保护区存在动物群落结构的差异。本调查初步掌握了四姑娘山保护区 内大中型兽类和林下活动鸟类的种类组成和相对多度。
\end{abstract}

关键词: 自然保护区; 红外相机; 生物多样性编目; 野生动物监测; 横断山脉

\section{Preliminary survey on mammal and bird diversity at Siguniang Mountains National Nature Reserve, Sichuan, China}

\author{
Xixi Hu${ }^{1}$, Weichao Zheng ${ }^{2}$, Jiaqi $\mathrm{Li}^{3}$, Sheng $\mathrm{Li}^{4}$, Han Yang ${ }^{5}$, Xing Chen ${ }^{1}$, Tianpei Guan ${ }^{*}$ \\ 1 Ecological Security and Protection Key Laboratory of Sichuan Province, Mianyang Teachers' College, Mianyang, \\ Sichuan 621000 \\ 2 Tangjiahe National Nature Reserve, Guangyuan, Sichuan 628100 \\ 3 Nanjing Institute of Environmental Sciences, Ministry of Ecology and Environment of the People's Republic of China, \\ Nanjing 210042 \\ 4 School of Life Sciences, Peking University, Beijing 100871 \\ 5 Siguniang Mountains National Nature Reserve, Aba, Sichuan 624200
}

\begin{abstract}
Knowledge of the abundance and distribution of biodiversity is vitally important to understand ecological processes, manage for conservation goals, and assess the impacts of global change. In order to investigate the diversity, abundance, and distribution of mammals and birds in Siguniang Mountains National Nature Reserve, we deployed 60 camera traps from May to December 2017. With an extensive survey effort of 11,013 camera-days, we recorded 31 mammal and bird species belonging to 7 orders and 18 families. Among the recorded species, five are first class and ten are second class national protected wildlife, whereas two are listed as Endangered, five as Vulnerable, and three as Near Threatened by the IUCN Red List. In addition to providing a preliminary inventory of large mammals and birds in the reserve, we also assessed the abundance of each species using the relative abundance index. According to this index, the five most abundant mammal species were tufted deer (Elaphodus cephalophus), sambar (Rusa unicolor), Himalayan marmot (Marmota himalayana), wild boar (Sus scrofa) and northern hog badger (Arctonyx collaris). The five most abundant avian species were blood pheasant (Ithaginis cruentus), white eared pheasant (Crossoptilon crossoptilon), giant laughing thrush (Garrulax maximus), chestnut-throated partridge (Tetraophasis obscurus)
\end{abstract}


and chestnut thrush (Turdus rubrocanus). Comparison between Siguniang Mountains and the adjacent Wolong Nature Reserve revealed different community compositions, this was likely due to distinct differences in local climate, vegetation, and topography between the two regions. The data obtained in this study will provide an important basis for future wildlife research and conservation management, and also provides support for biodiversity monitoring and regional inventories of mammals and birds.

Key words: nature reserve; camera-trapping; biodiversity inventory; wildlife monitoring; Hengduan Mountains

生物多样性调查与监测是生物多样性保护的 基础工作和重要手段(徐海根等, 2012)。自然保护区 网络是开展生物多样性调查与监测的重要基础(施 小刚等, 2017)。以往对自然保护区野生动物资源的 监测主要采用大样方调查法(张常智和张明海, 2011)、样线法(Stephens et al, 2006)和样带法(Harris et al, 1992)等。与传统野生动物调查方法相比, 红外 相机技术因其具有长期性、隐蔽性、准确性、非损 伤性等特点, 自20世纪90年代起开始被广泛应用于 野生动物研究(Karanth, 1995; O’Connell et al, 2011; Niedballa et al, 2015)。近年来, 红外相机技术在野生 动物本底资源调查(宋大昭等, 2014; 肖文宏等, 2014)、种群密度评估(Feng et al, 2001; Wang et al, 2014)、动物行为学研究(赵玉泽等, 2013; 薛亚东等, 2014)、生物多样性监测与保护管理(Wang et al, 2006; Li et al, 2012)等领域发挥了重要的作用。

利用红外相机技术对自然保护区的野生动物 本底资源进行监测和调查, 是促进自然保护区生物 多样性保护与研究的重要基础。四姑娘山国家级自 然保护区(以下简称四姑娘山保护区) 位于我国西南 山地, 其所处的横断山脉被认为是世界上高山植被 区系最丰富的地区和生物多样性分布中心之一(王 勇等, 2014)。虽然保护区建立至今已逾20年, 但除 本底资源调查外从未系统开展过针对兽类和鸟类 多样性的调查, 相关资料溃乏。为此, 我们利用红 外相机对四姑娘山的鸟兽多样性进行了初步调查, 旨在为四姑娘山生物多样性评价、保护管理和科学 研究提供基础数据。

\section{研究方法}

\section{1 研究区域}

四姑娘山保护区位于四川省阿坝州小金县, 与 卧龙国家级自然保护区、米亚罗自然保护区毗连, 地处横断山脉北段向四川盆地过渡的边缘地带, 介 于 $30^{\circ} 50^{\prime}-31^{\circ} 40^{\prime} \mathrm{N}$ 和 $102^{\circ} 00^{\prime}-103^{\circ} 30^{\prime} \mathrm{E}$ 之间, 属典
型的高山峡谷地貌, 其最高峰海拔 $6,250 \mathrm{~m}$ 。四姑娘 山地区属中亚热带季风气候向大陆性高原气候过 渡地区, 年降水量为600-1,400 mm, 该地区东南坡 的气象观测资料表明, 海拔1,120-4,500 $\mathrm{m}$ 最冷月气 温递减率为 $0.42{ }^{\circ} \mathrm{C} / 100 \mathrm{~m}$, 最暖月气温递减率为 $0.50^{\circ} \mathrm{C} / 100 \mathrm{~m}$, 气候垂直分异明显。区内生态条件复 杂, 生物群落类型多样, 植被垂直带谱明显, 物种 丰富, 其植物区系为喜马拉雅-横断山系的植物物 种(张启东等, 2013)。随海拔升高植被类型依次为: 沙棘(Hippophae rhamnoides)及稀疏灌从等半干旱 河谷植被以及农耕植被、落叶阔叶混交林、山地常 绿针叶林(暗针叶林)、亚高山灌丛草甸带、高山灌 从草甸带、高山草甸带、高山流石滩稀疏植被带。 海拔 5,400 $\mathrm{m}$ 以上为高山冻原一永冻带, 基本上无 植被分布(王勇等, 2014)。根据《四姑娘山植物资源 本底调查(2001)》, 四姑娘山是典型的高山草甸动物 类群分布区, 动物种类丰富, 垂直分异十分明显, 已发现脊椎动物 54 科 270 多种, 已确认兽类 46 种, 隶属于7目20科; 鸟类74种, 隶属 3 目 27 科。

\section{2 红外相机布设方案}

首先利用ArcGIS 10.2 将四姑娘山保护区划分 为 $1 \mathrm{~km} \times 1 \mathrm{~km}$ 的公里网格(图1), 再结合当地地形 和沟系走向、工作人员安置和维护相机的可达性, 以及野生动物出现频率等因素, 选择双桥沟、长坪 沟和海子沟三条主沟两侧坡面设置调查网格。每条 沟设置20个连续的公里网格, 在每个网格中心区域 放置1台红外相机。相机放置位置一般选择在兽径、 动物粪便或足迹遗留处、取食地以及水源附近等动 物经常活动和视野开阔的地区, 两个公里网格间红 外相机的距离不少于 $500 \mathrm{~m}$ (李佳琦等, 2018)。

为获取较高的相机工作效率, 放置相机主要遵 循以下两个原则: (1)红外相机主要固定在离地面约 50-80 cm的树上, 相机镜头避开阳光直射(例如朝 向西北方向), 并清理拍摄视野范围内过高或潜在 生长过高的植物(Swann et al, 2004), 以防止正午地 


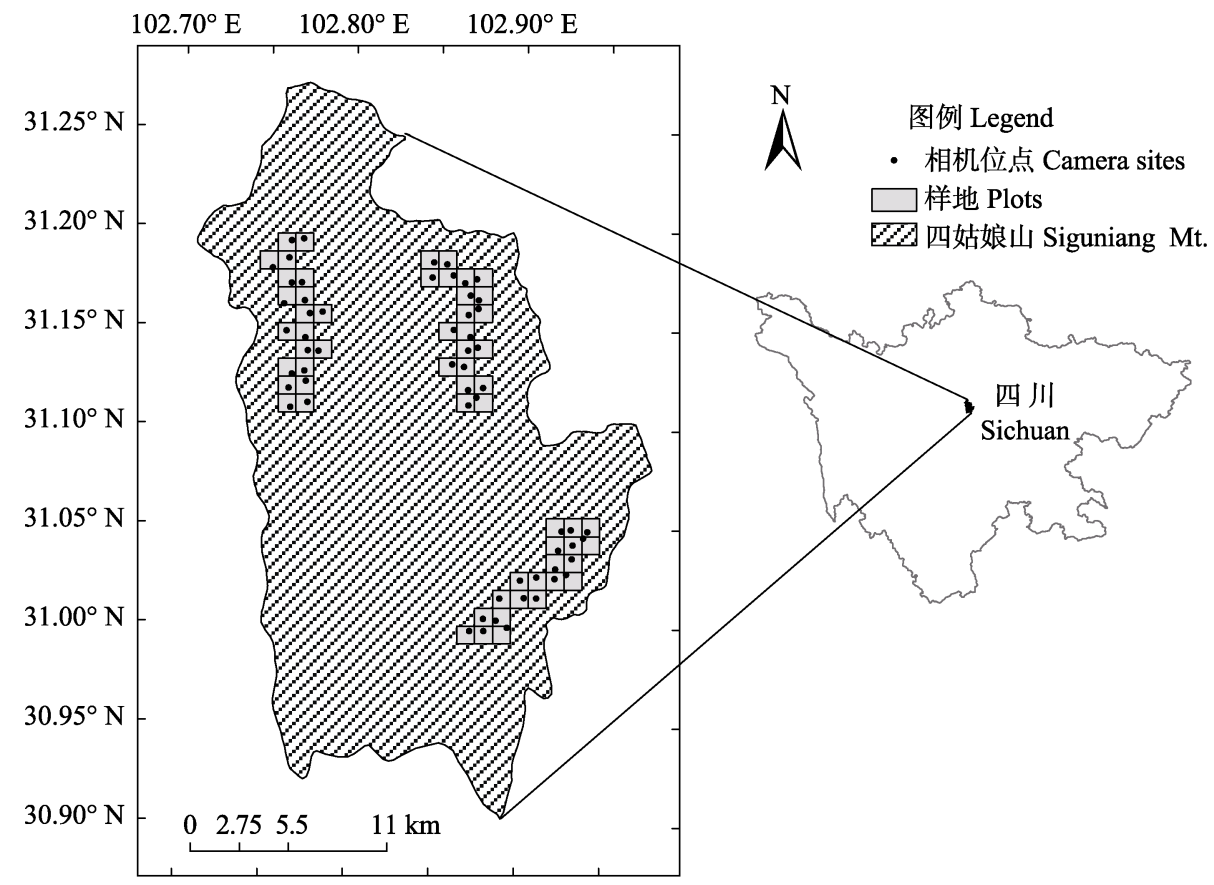

图1 四川省四姑娘山国家级自然保护区红外相机调查网格分布图

Fig. 1 Map of the camera-trapping survey blocks in Siguniang Mountains National Nature Reserve, Sichuan Province, China

面反射的光线过强或者镜头前新生植物反射光线 造成误拍(刘芳等, 2012); (2)相机镜头与地面呈小于 $10^{\circ}$ 的俯角, 以扩大相机拍摄的视野范围。在相机前 1-2 m视野开阔处放置嗅味剂(Carman's MagnaGlan Lure, PA, USA), 以增加路过的食肉动物在镜 头前的停留时间(施小刚等, 2017), 从而降低漏拍 率。安装好相机后, 对每个公里网格中的相机进行 编号(如51003F25A01即为四姑娘山保护区F25公里 网格中的相机), 并记录附近生境信息(相机编号、布 设时间、经纬度、海拔、生境类型及布设人等信息), 地形复杂的地区沿途做标记, 以便维护相机时更易 寻找。

2017年5-12月, 在四姑娘山的三条主要沟系共 布设了 60 台相机。相机型号为易安卫士L710, 安装 有8节电池以及 $16 \mathrm{G}$ 高速卡。相机参数设置为: 灵敏 度 “中”, 拍摄模式为“照片 + 视频”, 即连拍 3 张照 片，再录制一段10 s 的视频。

\section{3 数据处理与分析}

相机数据回收后删除误拍及无法识别的照片 和视频再进行物种鉴定。物种鉴定主要参考《中国 兽类野外手册》(Smith和解炎, 2009) 和《中国鸟类 野外手册》(约翰.马敬能等, 2000), 国家重点保护等 级参考《国家重点保护野生动物名录》 (http://www.forestry.gov.cn/main/3954/content-1063883. html), IUCN 濒危等级参考 IUCN 红色名录 (https: //www.iucnredlist.org/)。

完成物种鉴定后，将单台红外相机在野外持续 工作24 hi己为1个相机工作日(李晟等, 2016)。同一相 机在30 min内连续多次拍到的同一物种的所有图像 只记为 1 份独立有效照片(于桂清等, 2018)。每台相 机有效工作日以该位点拍摄到的第1张野外工作照 片(可能是工作人员或空拍照片)和最后 1 张野外工 作照片的日期间隔为有效工作日数。

使用相对多度指数 (relative abundance index, RAI)作为评估物种相对种群数量的指标(李勤等, 2013; 李晟等, 2014)。RAI以红外相机调查中同一物 种的拍摄率为基础，计算方法为:

$\mathrm{RAI}=($ 有效探测数 $/$ 总有效相机工作日 $) \times 1,000$

\section{2 结果}

60 台红外相机累计监测 11,013 个相机工作日, 单个相机位点的平均有效工作日为 184 天。共拍摄 到67,362张照片(视频也被归为照片处理)，可以明 确鉴定出物种的有效照片为 22,306 张，其中兽类 13,546 张 (60.73\%)，鸟类533张 (2.39\%), 工作人员 944 张，干扰(家畜、其他人员) 7,283 张，得到的独立 
有效照片为 4,517 张(附录1, 附录2)。

本次调查共记录到31种兽类和鸟类，隶属7目 18科(附录3), 包括5种国家一级重点保护野生动物 和10种国家二级重点保护野生动物。其中, 被IUCN 红色名录评估为濒危(EN)、易危(VU)和近危(NT) 的野生动物分别有 2 种、 5 种和 3 种。

\section{1 兽类组成}

调查期间，红外相机共拍摄到兽类18种，隶属 5目11科(附录3), 家畜及一些无法识别的鼠形小兽 并未记录在此物种名录中。18种兽类中, 偶蹄目最 多，共 4 科 8 种，占兽类总物种数的 $44.44 \%$; 其次为 食肉目，共 4 科 7 种，占兽类总物种数的 $38.89 \%$; 啮 齿目、灵长目和兔形目最少, 均只有1种, 分别仅占 兽类总物种数的 $5.56 \%$ 。

本次调查共记录到国家一级重点保护野生动 物 3 种, 即林鹰 (Moschus berezovskii)、马 鹿 (M. chrysogaster)、雪豹 (Panthera uncia); 国家二级重点 保护野生动物7种: 亚洲黑熊(Ursus thibetanus)、中 icolor)、岩羊(Pseudois nayaur)、猕猴(Macaca mulatta)、中华斑羚 (Naemorhedus griseus)、黄喉貂 (Martes flavigula)。林鹰和马疴被IUCN红色名录列 为濒危物种 $(E N)$; 雪豹、亚洲黑熊、中华斑羚和水 鹿等4种被列为易危物种(VU); 毛冠鹿(Elaphodus cephalophus)和中华镾羚被列为近危物种(NT)。

此次调查发现种群较丰富的兽类为毛冠鹿 $(R A I=145.92)$ 、水鹿 $(R A I=33.32)$ 、喜马拉雅旱獭 (Marmota himalayana, RAI = 21.43)、野 (Sus scrofa, $\mathrm{RAI}=19.61) 、$ 猪獾 $($ Arctony $x$ collaris, RAI = 18.34 $)$, 它们的相对多度指数和拍摄率均居于兽类前五位。 在60个网格中, 分布最广泛的野生兽类依次是毛冠 鹿 $(n=54)$ 、中华瓺羚 $(n=36)$ 、野猪 $(n=35)$ 、水鹿 $(n=32)$ 和猪獾 $(n=32)$ 。偶蹄目是调查区域兽类中 多度最高的类群, 在相对多度指数、拍摄率和拍摄 位点数中居前五位的分别有 3 个、 3 个和 4 个物种。 而此次监测到的食肉目 (7种)的物种数虽然只比偶 蹄目(8种)少 1 种, 但除了猪獾以外相对多度指数和 拍摄率都较低, 拍摄位点也较少, 其中大型的食肉 动物雪豹 $(\mathrm{RAI}=0.09)$ 和狼 (Canis lupus, RAI $=0.09)$ 均仅在1个相机位点上记录到1次。

\section{2 乌类组成}

本次调查共记录到13种鸟类, 隶属 2 目7科(附

录 3), 雀形目最多, 共 6 科 8 种, 占鸟类种数的 $61.54 \%$ 。鸡形目次之, 共1科 5 种, 占鸟类种数的 $38.46 \%$ 。本调查共记录到国家一级重点保护鸟类2 种: 绿尾虹雉 (Lophophorus lhuysii)和雉㝇 (Tetraophasis obscurus); 国家二级重点保护野生动物 3 种: 藏雪鸡(Tetraogallus tibetanus)、血雉(Ithaginis cruentus)和白马鸡(Crossoptilon crossoptilon)。绿尾虹雉 被IUCN红色名录列为易危物种 (VU), 白马鸡列为 近危物种(NT)。

调查结果显示, 多度最高的鸟类分别是血雉 $(\mathrm{RAI}=5.81)$ 、白马鸡 $(\mathrm{RAI}=2.81)$ 、大噪鹃 (Garrulax maximus) $(\mathrm{RAI}=1.91)$ 、雉敦 $(\mathrm{RAI}=1.09)$ 和灰头鸫 (Turdus rubrocanus) (RAI $=0.54)$, 它们的相对多度 指数和拍摄率均位列前五。在调查区域中, 分布最 广的鸟类依次是血雉(18个)、白马鸡 (9个)、灰头鸫 (4个)和大噪鸹 $(4$ 个), 其余鸟类的拍摄位点均为 2 个 或1个。相较于兽类, 此次调查记录到的鸟类的相对 丰富度更低, 拍摄位点以及物种数也较少。

\section{3 讨论}

本次调查监测到的兽类和鸟类物种数占该区 域历史记载的兽类和鸟类物种数的 $36.97 \%$ 和 $17.57 \%$ 。这种情况一方面可能是由于兽类中的啮齿 目、兔形目、劳亚食虫目个体较小, 无法通过红外 相机进行有效记录和准确识别所致。另一方面，鸟 类中的非地栖种类也无法通过红外相机获取足够 的信息。加之调查位点数量和时间的局限，以及可 能存在的历史数据错误, 导致本次调查记录的物种 仅占资料记载的少部分。因此, 要全面评估保护区 内的动物物种多样性, 在加强调查力度的同时还需 要其他调查手段的辅助。

四姑娘山位于邛崃山系的西侧，与东面的卧龙 国家级自然保护区以及北面的米亚罗自然保护区 共同构成了该山系的保护区网络核心。但四姑娘山 在物种组成上与卧龙自然保护区(施小刚等, 2017) 相比还存在较大的差异, 此次调查并未拍摄到卧龙 保护区的旗舰物种大熊猫(Ailuropoda melanoleuca) 及羚牛(Budorcas taxicolor)。我们认为这可能是由于 四姑娘山保护区起始海拔偏高(大于 $3,000 \mathrm{~m}$ ), 缺乏 大熊猫赖以生存的竹类资源, 再加上低海拔段栖息 地缺乏导致具有迁移习性的动物(例如羚牛, Guan et al, 2013)无法完成整个生活过程。因此, 本调查也 
体现了栖息地的多样性水平是限制物种多样性的 重要因素(铁军等, 2015)。

在本次调查中记录到了处于食物链顶端的以 食肉为主的 2 种大型食肉动物, 即雪豹和狼。它们的 相对多度都很低，都仅在 1 个相机位点记录到 1 次， 说明四姑娘山保护区内大中型食肉动物的种群数 量和密度可能处于较低水平。雪豹等大型食肉动物 可控制中层捕食者与食草动物数量, 能维持高山生 态系统的稳定(Gordon et al, 2015), 但其需要大范围 的活动空间来提供充足的食物(Ripple et al, 2013; 蒋志刚等, 2015)。随着四姑娘山地区旅游业的开发 和人类活动海拔的逐渐升高(放牧、砍伐、采药等), 雪豹栖息地面积逐渐减小并呈现破碎化, 将导致种 群分布受到进一步挤压。因此, 保护好雪豹等大型 食肉动物还依赖于降低栖息地干扰。另外, 雪豹主 要分布在林线以上的灌从、流石滩区域(Jackson, 1996; Gordon et al, 2015), 本次调查布设的红外相 机位点仅有少数位于雪豹的分布海拔段, 也是仅一 个位点拍摄到雪豹的原因。因此, 为了全面掌握雪 豹的种群现状及分布, 增强高海拔段的红外监测力 度是很有必要的。充足的有蹄类种群是维持食肉动 物种群规模的重要基础(Buzzard et al, 2017)。偶蹄 类是在四姑娘山监测到的相对多度指数和拍摄位 点最多的类群, 特别是毛冠鹿、水鹿、野猪和中华 䯷羚。因此, 丰富的有蹄类种类和可观的种群数量 对维持一定规模的食肉动物是有利的(Ripple et al, 2014; Gordon et al, 2015; 施小刚等, 2017)。

调查还发现四姑娘山存在较强的人类干扰, 主 要是家养动物(包括牛、马、家犬), 约 $26 \%$ 的有效照 片是因放牧而误拍的。值得注意的是, 我们在多个 观测位置发现野生动物与家养动物存在栖息地重 叠。家畜会在栖息地空间、食物和水源等资源上与 野生动物产生竞争 (洪起平等, 2007; 李佳等, 2018)。成群家畜的活动不仅会影响野生动物的分布 和繁衍(Dublin et al, 1990; Scognamillo et al, 2003), 其所携带的病毒、细菌等病原体也会给野生动物种 群带来严重的威胁(Pelletier, 2006; 吴问国，2008; 施小刚等, 2017)。

基于自然保护区网络的生物多样性调查与监 测, 能及时掌握我国生物多样性的现状、动态变化 和面临的威胁, 为区域性和全国性生物多样性保护 策略的制定奠定基础。本次调查是对四姑娘山进行
的第一次基于红外相机技术的大中型兽类和鸟类 的多样性水平评估，调查结果初步反映了四姑娘山 野生动物群落的结构和物种相对多度, 发现四姑娘 山拥有较高的动物多样性, 是多个国家重点保护动 物的分布区。然而，由于四姑娘山存在较强的旅游 带来的干扰，保护区还应加强对干扰源的管控，适 当限制保护区内的放牧活动，减少当地居民及家畜 进入重点保护物种的核心分布区和降低人类活动 对生物多样性的影响。

致谢: 本次调查获得了四姑娘山国家级自然保护区 管理局的大力支持以及卧龙国家级自然保护区的 协助，卧龙沐江坪保护站的胡强、曾永兵、曾国庆 等参加了红外相机的安装与维护工作, 在此一并致 谢!

\section{参考文献}

Buzzard PJ, Li XY, Bleisch W (2017) The status of snow leopards Panthera uncia, and high altitude use by common leopards $P$. pardus, in north-west Yunnan, China. Oryx, 51, 587-589.

Dublin HT, Sinclair ARE, Boutin S, Anderson E, Jago M, Arcese P (1990) Does competition regulate ungulate populations? Further evidence from Serengeti, Tanzania. Oecologia, 82, 283-288.

Feng LM, Wang TM, Mou P, Kou XJ, Ge JP (2001) First image of an Amur leopard recorded in China. Cat News, 55, 9.

Gordon CE, Feit A, Gurber J, Letnic M (2015) Mesopredator suppression by an apex predator alleviates the risk of predation perceived by small prey. Proceedings of the Royal Society B: Biological Sciences, 282, 20142870.

Guan TP, Ge BM, McShea WJ, Li S, Song YL (2013) Seasonal migration by a large forest ungulate: A study on takin (Budorcas taxicolor) in Sichuan Province, China. European Journal of Wildlife Research, 59, 81-91.

Harris RB, Liu YS, Cai GQ, Bart WO (1992) Line transects for estimating density of musk deer pellet groups. Acta Theriologica Sinica, 12, 302-305. (in Chinese) [Harris RB, 刘永生, 蔡桂全, Bart WO (1992) 使用样线法进行马看粪 堆密度的测量. 兽类学报, 12, 302-305.]

Hong QP, Ding P, Ding BY (2007) Investigation and Research of Nature Resources in Fengyang Mountains. China Forestry Publishing House, Beijing. (in Chinese) [洪起平, 丁平, 丁 炳扬 (2007) 凤阳山自然资源考察与研究. 中国林业出 版社, 北京.]

Jackson RM (1996) Home Range, Movements and Habitat Use of Snow Leopard (Uncia uncia) in Nepal. PhD dissertation, University of London, London.

Jiang ZG, Ma Y, Wu Y, Wang YX, Feng ZJ, Zhou KY, Liu 
SY, Luo ZH, Li CW (2015) China’s mammalian diversity. Biodiversity Science, 23, 351-364. (in Chinese with English abstract) [蒋志刚, 马勇, 吴毅, 王应祥, 冯祚建, 周开亚, 刘少英, 罗振华, 李春旺 (2015) 中国哺乳动物多样性. 生物多样性, 23, 351-364.]

Karanth KU (1995) Estimating tiger Panthera tigris populations from camera-trap data using capture-recapture models. Biological Conservation, 71, 333-338.

Li J, Liu F, Ye LX, Liu SL, Peng H, Li DQ (2018) Camera-trapping survey of the diversity of mammals and birds in Fengyang Mountain of Zhejiang Province, China. Acta Theriologica Sinica, 38, 95-103. (in Chinese with English abstract) [李佳, 刘芳, 叶立新, 刘胜龙, 彭辉, 李 迪强 (2018) 利用红外相机调查浙江省凤阳山兽类和鸟 类多样性. 兽类学报, 38, 95-103.]

Li JQ, Xu HG, Wan YQ, Sun JX, Li S, Cai L (2018) Progress in construction of China Mammal Diversity Observation Network (China BON-Mammals). Journal of Ecology and Rural Environment, 34, 12-19. (in Chinese with English abstract) [李佳琦, 徐海根, 万雅琼, 孙佳欣, 李晟, 蔡蕾 (2018) 全国哺乳动物多样性观测网络 (China BON-Mammals) 建设进展. 生态与农村环境学报, 34, 12-19.]

Li Q, Wu JG, Kou XJ, Feng LM (2013) Applications of camera trap in wildlife population ecology. Chinese Journal of Applied Ecology, 24, 947-955. (in Chinese with English abstract) [李勤, 邬建国, 寇晓军, 冯利民 (2013) 相机陷 阱在野生动物种群生态学中的应用. 应用生态学报, 24, 947-955.]

Li S, McShea WJ, Wang D, Lu Z, Gu X (2012) Gauging the impact of management expertise on the distribution of large mammals across protected areas. Diversity and Distributions, 18, 1166-1176.

Li S, Wang DJ, Bu HL, Liu XG, Jin T (2016) Camera-trapping survey on the mammal diversity of the Laohegou Nature Reserve, Sichuan Province. Acta Theriologica Sinica, 36, 282-291. (in Chinese with English abstract) [李晟, 王大军, 卜红亮, 刘小庚, 靳粀 (2016) 四川省老河沟自然保护区 兽类多样性红外相机调查. 兽类学报, 36, 282-291.]

Li S, Wang DJ, Xiao ZS, Li XH, Wang TM, Feng LM, Wang $Y$ (2014) Camera-trapping in wildlife research and conservation in China: Review and outlook. Biodiversity Science, 22, 685-695. (in Chinese with English abstract) [李 晟, 王大军, 肖治术, 李欣海, 王天明, 冯利民, 王云 (2014) 红外相机技术在我国野生动物研究与保护中的应 用与前景. 生物多样性, 22, 685-695.]

Liu F, Li DQ, Wu JG (2012) Using infra-red cameras to survey wildlife in Beijing Songshan National Nature Reserve. Acta Ecologica Sinica, 32, 730-739. (in Chinese with English abstract) [刘芳, 李迪强, 吴记贵 (2012) 利用红外相机调 查北京松山国家级自然保护区的野生动物物种. 生态学 报, 32, 730-739.]

Mackinnon J, Phillipps K, He FQ (2000) A Field Guide to the Birds of China. Hunan Education Press, Changsha. (in Chinese) [约翰·马敬能, 卡伦·菲利普斯, 何芬奇 (2000)
中国鸟类野外手册. 湖南教育出版社, 长沙.]

Niedballa J, Sollmann R, Mohamed AB, Bender J, Wilting A (2015) Defining habitat covariates in camera-trap based occupancy studies. Scientific Reports, 5, 17041.

O’Connell AF, Nichols JD, Karanth KU (2011) Camera Traps in Animal Ecology: Methods and Analyses. Springer, New York.

Pelletier F (2006) Effects of tourist activities on ungulate behavior in a mountain protected area. Journal of Mountain Ecology, 8, 5-19.

Ripple WJ, Estes JA, Beschta RL, Wilmers CC, Ritchie EG, Hebblewhite M, Berger J, Elmhagen B, Letnic M, Nelson MP, Schmitz OJ, Smith DW, Wallach AD, Wirsing AJ (2014) Status and ecological effects of the world's largest carnivores. Science, 343, 151.

Ripple WJ, Wirsing AJ, Wilmers CC, Letnic M (2013) Wide spread mesopredator effects after wolf extirpation. Biological Conservation, 160, 70-79.

Scognamillo D, Maxit IE, Sunquist M, Polisar J (2003) Coexistence of jaguar (Panthera onca) and puma (Puma concolor) in a mosaic landscape in the Venezuelan llanos. Journal of Zoology, 259, 269-279.

Shi XG, Hu Q, Li JQ, Tang Z, Yang J, Li WJ, Shen XL, Li S (2017) Camera-trapping surveys of the mammal and bird diversity in Wolong National Nature Reserve, Sichuan Province. Biodiversity Science, 25, 1131-1136. (in Chinese with English abstract) [施小刚, 胡强, 李佳琦, 唐卓, 杨 建, 李文静, 申小莉, 李晟 (2017) 利用红外相机调查四 川卧龙国家级自然保护区鸟兽多样性. 生物多样性, 25, 1131-1136.]

Smith AT, Xie Y (2009) A Guide to the Mammals of China. Hunan Education Press, Changsha. (in Chinese) [Smith AT, 解炎 (2009) 中国兽类野外手册. 湖南教育出版社, 长沙.]

Song DZ, Wang BP, Jiang JY, Wan SP, Cui SM, Wang TM, Feng LM (2014) Using camera trap to monitor a North Chinese leopard (Panthera pardus japonesis) population and their main ungulate prey. Biodiversity Science, 22, 733-736. (in Chinese with English abstract) [宋大昭, 王卜平, 蒋进 原，万绍平，崔士明，王天明，冯利民（2014）山西晋中 庆城林场华北豹及其主要猎物种群的红外相机监测. 生 物多样性, 22, 733-736.]

Stephens PA, Zaumyslova OY, Miquelle DG, Myslenkov AI, Hayward GD (2006) Estimating population density from indirect sign: Track counts and the Formozov-MalyshevPereleshin formula. Animal Conservation, 9, 339-348.

Swann DE, Hass CC, Dalton DC, Wolf SA (2004) Infraredtriggered cameras for detecting wildlife: An evaluation and review. Wildlife Society Bulletin, 32, 357-365.

Tie J, Li YF, Wang X, Bai FL, Cui FT, Jin S (2015) Species diversity of forest community in Macaca mulatta habitat in Lishan National Nature Reserve, Shanxi. Chinese Journal of Ecology, 34, 3009-3015. (in Chinese with English abstract) [铁军, 李燕芬, 王霞, 白凤麟, 崔方天, 金山 (2015) 山 西历山国家级自然保护区猕猴栖息地森林群落物种多样 性. 生态学杂志, 34, 3009-3015.] 
Wang DJ, Li S, McShea WJ, Li MF (2006) Use of remote-trip cameras for wildlife surveys and evaluating the effectiveness of conservation activities at a nature reserve in Sichuan Province, China. Environmental Management, 38, 942-951.

Wang Y, Wang YD, Tao SC, Chen XP, Kong YP, Asif S, Ye CY, Pang M (2014) Using infra-red camera trapping technology to monitor mammals along Karakorum Highway in Khunjerab National Park, Pakistan. Pakistan Journal of Zoology, 46, 725-731.

Wang Y, Zhang QD, Yang H (2014) Study on water conservation capacity of three typical vegetation types in Siguniang Mountain National Natural Reserve. Sichuan Environment, 33(5), 111-114. (in Chinese with English abstract) [王勇, 张启东, 杨晗 (2014) 四姑娘山国家级自 然保护区3种典型植被类型水源涵养功能研究. 四川环 境, 33(5), 111-114.]

Wu WG (2008) Current situation and management countermeasure of Jiangxi Taohongling Sika Deer National Nature Reserve. Sichuan Journal of Zoology, 27, 457-459. (in Chinese with English abstract) [吴问国 (2008) 江西桃红 岭野生梅花鹿保护现状及管理对策. 四川动物, 27 , 457-459.]

Xiao WH, Feng LM, Zhao XD, Yang HT, Dou HL, Cheng YC, Mou P, Wang TM, Ge JP (2014) Distribution and abundance of Amur tiger, Amur leopard and their ungulate prey in Hunchun National Nature Reserve, Jilin. Biodiversity Science, 22, 717-724. (in Chinese with English abstract) [肖 文宏, 冯利民, 赵小丹, 杨海涛, 窦海龙, 程艳超, 牟溥, 王天明, 葛剑平 (2014) 吉林珲春自然保护区东北虎和 东北豹及其有蹄类猎物的多度与分布. 生物多样性, 22, 717-724.]

Xu HG, Ding H, Wu J, Cao MC, Chen L, Le ZF, Cui P (2012) Interpretation of the 2020 global biodiversity targets and its assessment indicators. Journal of Ecology and Rural Environment, 28, 1-9. (in Chinese with English abstract) [徐海根, 丁晖, 吴军, 曹铭昌, 陈炼, 乐志芳, 崔鹏 (2012) 2020年全球生物多样性目标解读及其评估指标探 讨. 生态与农村环境学报, 28, 1-9.]
Xue YD, Liu F, Guo TZ, Yuan L, Li DQ (2014) Using camera traps to survey wildlife at water sources on the northern slope of the Altun Mountains, China. Acta Theriologica Sinica, 34, 164-171. (in Chinese with English abstract) [薛 亚东，刘芳，郭铁征，袁磊，李迪强（2014）基于相机陷 阱技术的阿尔金山北坡水源地鸟兽物种监测. 兽类学报, 34, 164-171.]

Yu GQ, Kang ZJ, Liu MS, Chen ZF, Deng ZC (2018) Preliminary survey using infrared camera reveals fauna and avifauna diversity at Hupingshan National Nature Reserve, Hunan, China. Acta Theriologica Sinica, 38, 104-112. (in Chinese with English abstract) [于桂清, 康祖杰, 刘美斯, 陈振法, 邓忠次 (2018) 利用红外相机对湖南壶瓶山国 家级自然保护区兽类和鸟类多样性的初步调查. 兽类学 报, 38, 104-112.]

Zhang CZ, Zhang MH (2011) Population status and dynamic trends of Amur tiger's prey in Eastern Wandashan Mountain, Heilongjiang Province. Acta Ecologica Sinica, 31, 6481-6487. (in Chinese with English abstract) [张常智, 张明海 (2011) 黑龙江省东完达山地区东北虎猎物种群 现状及动态趋势. 生态学报, 31, 6481-6487.]

Zhang QD, Xie Q, Yang H, Wang M, Xu W (2013) Study of water conservation capacity of Minjiang fir litter and soil of Siguniang Mountains National Natural Reserve. Sichuan Environment, 32(2), 42-45. (in Chinese with English abstract) [张启东, 谢强, 杨晗, 王蒙, 徐玮 (2013) 四姑 娘山国家级自然保护区岷江冷杉枯落物及土壤的持水能 力研究. 四川环境, 32(2), 42-45.]

Zhao YZ, Wang ZC, Xu JL, Luo X, An LD (2013) Activity rhythm and behavioral time budgets of wild Reeves's pheasant (Syrmaticus reevesii) using infrared camera. Acta Ecologica Sinica, 33, 6021-6027. (in Chinese with English abstract) [赵玉泽, 王志臣, 徐基良, 罗旭, 安丽丹 (2013) 利用红外照相技术分析野生白冠长尾雉活动节律及时间 分配. 生态学报, 33, 6021-6027.]

(责任编委: 丁平 责任编辑: 问文杰)

\section{附录 Supplementary Material}

\section{附录1 四姑娘山国家级自然保护区红外相机调查中记录到的兽类和鸟类物种统计记录总表}

Appendix 1 Summary table of mammal and bird species recorded by the camera-trapping survey in the Siguniang Mountains National Nature Reserve, Sichuan Province, China http://www.biodiversity-science.net/fileup/PDF/2018260-1.xlsx

\section{附录2 四姑娘山国家级自然保护区红外相机调查中记录到的代表物种}

Appendix 2 The representative species taken by camera-trapping survey in the Siguniang Mountains National Nature Reserve, Sichuan Province, China http://www.biodiversity-science.net/fileup/PDF/2018260-2.pdf

\section{附录3 四姑娘山国家级自然保护区红外相机调查中记录到的兽类和鸟类物种}

Appendix 3 Mammal and bird species recorded by the camera-trapping in the Siguniang Mountains National Nature Reserve, Sichuan Province, China 
胡茜茜, 郑维超, 李佳琦, 李晟, 杨晗, 陈星, 官天培. 四姑娘山国家级自然保护区鸟兽多样性初步调查. 生物多样性, 2018, 26 (12): 1325-1331.

http://www.biodiversity-science.net/CN/10.17520/biods.2018260

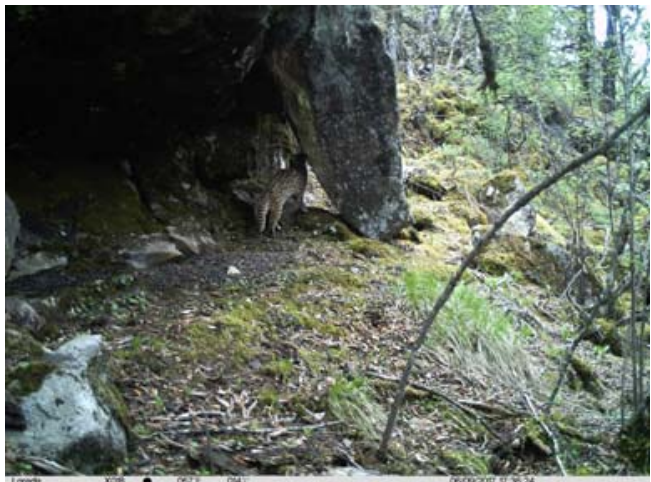

豹猫 Prionailurus bengalensis

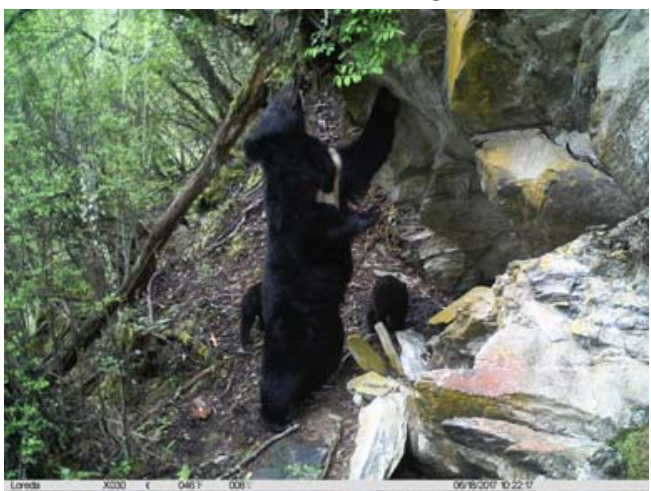

亚洲黑熊 Ursus thibetanus

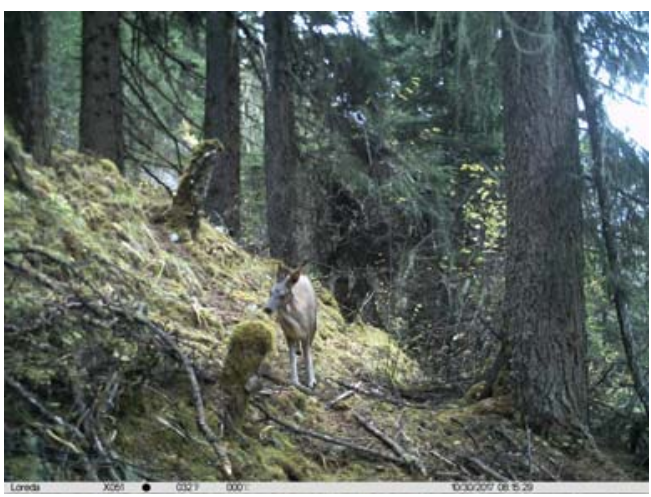

林鹿 Moschus berezovskii

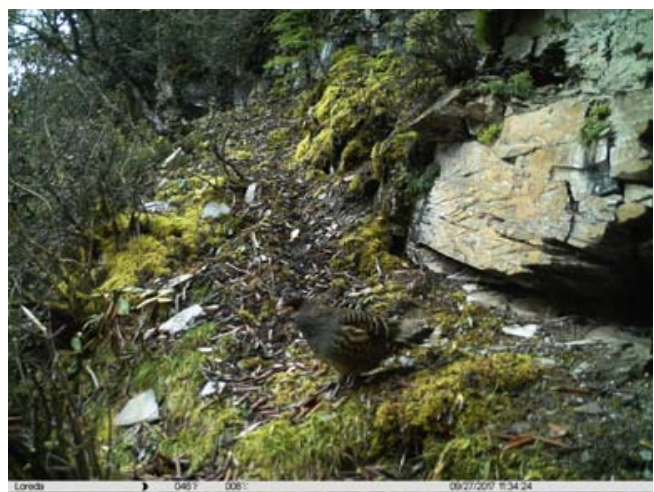

雉鹑 Tetraophasis obscures

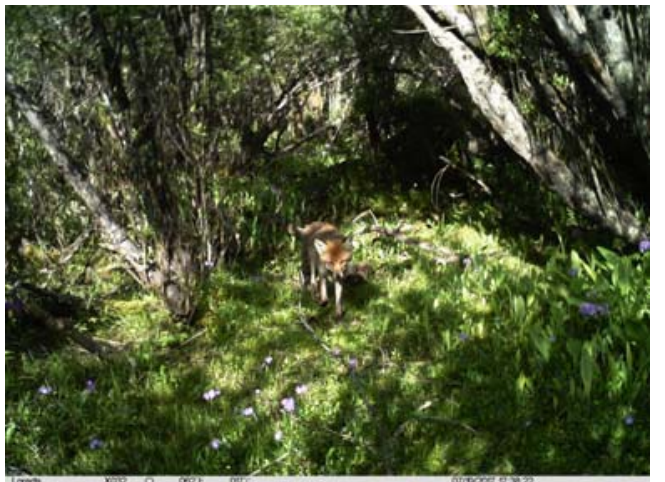

赤狐 Vulpes vulpes

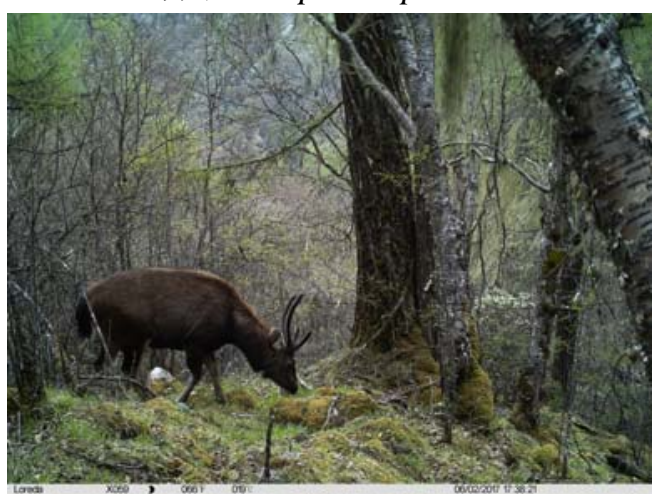

水鹿 Rusa unicolor

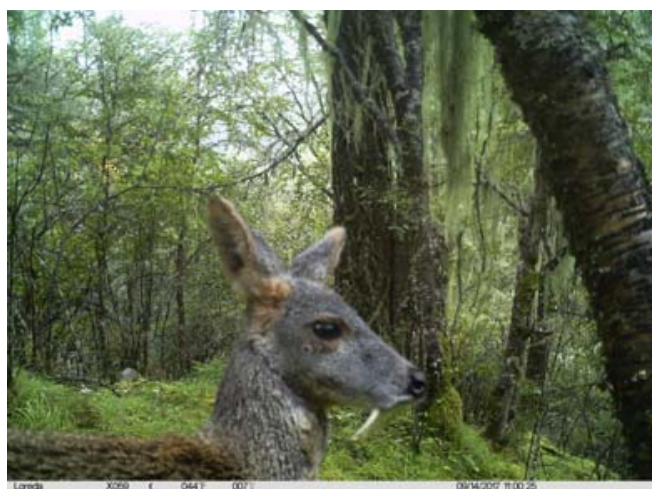

马㯍 Moschus chrysogaster

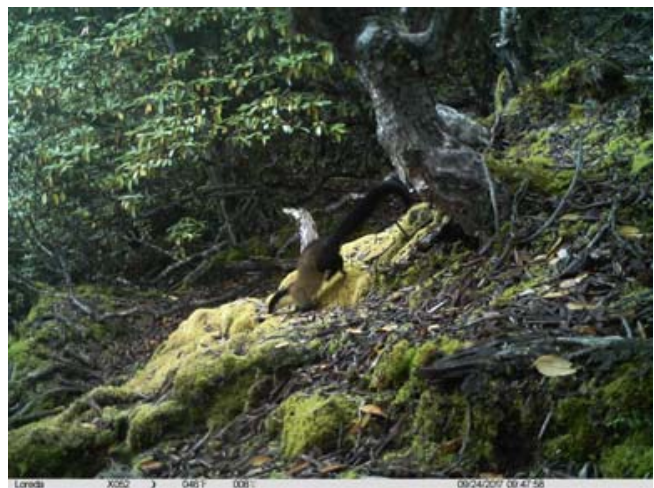

黄喉貂 Martes flavigula

附录2 四姑娘山国家级自然保护区红外相机调查中记录到的代表物种

Appendix 2 The representative species taken by camera-trapping survey in the Siguniang Mountains National Nature Reserve, Sichuan Province, China 
胡茜茜, 郑维超, 李佳琦, 李戟, 杨晗, 陈星, 官天培. 四姑娘山国家级自然保护区鸟兽多样性初步调查. 生 物多样性, 2018, 26 (12): 1325-1331.

http://www.biodiversity-science.net/CN/10.17520/biods.2018260

附录3 四姑娘山国家级自然保护区红外相机调查中记录到的兽类和鸟类物种

Appendix 3 Mammal and bird species recorded by the camera-trapping in the Siguniang Mountains National Nature Reserve, Sichuan Province, China

\begin{tabular}{|c|c|c|c|c|c|c|}
\hline 物种 Species & $\begin{array}{l}\text { 保护等级 } \\
\text { National } \\
\text { protected } \\
\text { category }\end{array}$ & $\begin{array}{c}\text { IUCN } \\
\text { 红色名录 } \\
\text { IUCN Red } \\
\text { List }\end{array}$ & $\begin{array}{c}\text { 独立有效照片 } \\
\text { 数 No. of } \\
\text { independent } \\
\text { photographs }\end{array}$ & $\begin{array}{c}\text { 拍到照片的 } \\
\text { 网格数 } \\
\text { No. of grids }\end{array}$ & $\begin{array}{c}\text { 相对多度指数 } \\
\text { Relative } \\
\text { abundance } \\
\text { index } \\
\text { (RAI) }\end{array}$ & $\begin{array}{c}\text { 海拔范围 } \\
\text { Elevation } \\
\text { range } \\
(\mathrm{m})\end{array}$ \\
\hline $\begin{array}{l}\text { 兽类 Mammals } \\
\text { 食肉目 Carnivora } \\
\text { 猫科 Felidae }\end{array}$ & & & & & & \\
\hline 雪豹 Panthera uncia & I & VU & 1 & 1 & 0.09 & 4,096 \\
\hline \multicolumn{7}{|l|}{ 犬科 Canidae } \\
\hline 狼 Canis lupus & & LC & 1 & 1 & 0.09 & 3,797 \\
\hline \multicolumn{7}{|l|}{ 熊科 Ursidae } \\
\hline \multicolumn{7}{|l|}{ 鼠由科 Mustelidae } \\
\hline 猪獾 Arctonyx albogularis & & LC & 202 & 32 & 18.34 & $3,490-4,219$ \\
\hline $\begin{array}{l}\text { 黄喉貂 Martes flavigula } \\
\text { 偶蹄目 Cetartiodactyla } \\
\text { 鹿科 Cervidae }\end{array}$ & II & $\mathrm{LC}$ & 13 & 9 & 1.18 & $3,511-4,011$ \\
\hline 水鹿 Rusa unicolor & II & VU & 367 & 32 & 33.32 & $3,490-4,203$ \\
\hline \multicolumn{7}{|l|}{ 牛科 Bovidae } \\
\hline 中华斑羚 Naemorhedus griseus & II & VU & 84 & 22 & 7.63 & $3,490-4,039$ \\
\hline 岩羊 Pseudois nayaur & II & LC & 118 & 6 & 10.71 & $3,781-4,203$ \\
\hline $\begin{array}{l}\text { 中华鬛羚 Capricornis milneedwardsii } \\
\text { 榭科 Moschidae }\end{array}$ & II & NT & 187 & 36 & 16.98 & $3,490-4,039$ \\
\hline 林謝 Moschus berezovskii & I & EN & 16 & 6 & 1.45 & $3,539-4,039$ \\
\hline \multicolumn{7}{|l|}{ 猪科 Suidae } \\
\hline 野猪 Sus scrofa & & LC & 216 & 35 & 19.61 & $3,511-4,039$ \\
\hline \multicolumn{7}{|l|}{ 啮齿目 Rodentia } \\
\hline 喜马拉雅旱獭 Marmota himalayana & & LC & 236 & 8 & 21.43 & $3,750-4,219$ \\
\hline \multicolumn{6}{|l|}{ 兔形目 Lagomorpha } & 兔科 Leporidae \\
\hline \multicolumn{7}{|l|}{ 灵长目 Primates } \\
\hline \multicolumn{7}{|l|}{ 猴科 Ceropithecidae } \\
\hline 猕猴 Macaca mulatta & II & $\mathrm{LC}$ & 14 & 8 & 1.21 & $3,490-3,816$ \\
\hline
\end{tabular}


胡茜茜, 郑维超, 李佳琦, 李晟, 杨晗, 陈星, 官天培. 四姑娘山国家级自然保护区鸟兽多样性初步调查. 生 物多样性, 2018, 26 (12): 1325-1331.

http://www.biodiversity-science.net/CN/10.17520/biods.2018260

\begin{tabular}{|c|c|c|c|c|c|c|}
\hline 物种 Species & $\begin{array}{l}\text { 保护等级 } \\
\text { National } \\
\text { protected } \\
\text { category }\end{array}$ & $\begin{array}{c}\text { IUCN } \\
\text { 红色名录 } \\
\text { IUCN Red } \\
\text { List }\end{array}$ & $\begin{array}{c}\text { 独立有效照片 } \\
\text { 数 No. of } \\
\text { independent } \\
\text { photographs }\end{array}$ & $\begin{array}{l}\text { 拍到照片的 } \\
\text { 网格数 } \\
\text { No. of grids }\end{array}$ & $\begin{array}{c}\text { 相对多度指数 } \\
\text { Relative } \\
\text { abundance } \\
\text { index } \\
\text { (RAI) }\end{array}$ & $\begin{array}{c}\text { 海拔范围 } \\
\text { Elevation } \\
\text { range } \\
\text { (m) }\end{array}$ \\
\hline \multicolumn{7}{|l|}{$\begin{array}{l}\text { 鸟类 Birds } \\
\text { 雀形目 Passeriformes } \\
\text { 冻科 Turdidae }\end{array}$} \\
\hline $\begin{array}{l}\text { 灰头鸫 Turdus rubrocanus } \\
\text { 画眉科 Timaliidae }\end{array}$ & \multicolumn{5}{|c|}{ 画眉科 Timaliidae } & $3,788-4,219$ \\
\hline $\begin{array}{l}\text { 大噪鹋 Garrulax maximus } \\
\text { 雀科 Paridae }\end{array}$ & \multicolumn{5}{|c|}{ 雀科 Paridae } & $3,536-4,039$ \\
\hline \multicolumn{7}{|l|}{ 暡科 Muscicapidae } \\
\hline 蓝额红尾鸲 Phoenicurus frontalis & & LC & 2 & 1 & 0.18 & 4,219 \\
\hline $\begin{array}{l}\text { 长尾地冻 Zoothera dixoni } \\
\text { 鸦科 Corvidae }\end{array}$ & & LC & 5 & 1 & 0.45 & 4,039 \\
\hline 大嘴乌鸦 Corvus macrorhynchos & & $\mathrm{LC}$ & 1 & 1 & 0.09 & 3,843 \\
\hline 红嘴山鸦 Pyrrhocorax pyrrhocorax & & LC & 3 & 1 & 0.27 & 4,027 \\
\hline \multicolumn{7}{|l|}{ 岩翏科 Prunellidea } \\
\hline 栗背岩翏 Prunella immaculata & & $\mathrm{LC}$ & 1 & 1 & 0.09 & 3,718 \\
\hline \multicolumn{2}{|l|}{ 雉科 Phasianidae } & & & & & \\
\hline 白马鸡 Crossoptilon crossoptilon & II & NT & 31 & 9 & 2.81 & $3,788-4,203$ \\
\hline 藏雪鸡 Tetraogallus tibetanus & II & $\mathrm{LC}$ & 2 & 2 & 0.18 & $3,718-4,219$ \\
\hline 血雉 Ithaginis cruentus & II & LC & 64 & 18 & 5.81 & $3,528-4,039$ \\
\hline 雉敦 Tetraophasis obscurus & I & LC & 12 & 2 & 1.09 & $3,948-4,027$ \\
\hline 绿尾虹雉 Lophophorus Ihuysii & I & VU & 2 & 2 & 0.18 & 4,027 \\
\hline \multicolumn{7}{|l|}{ 其他 Others } \\
\hline 家狗 Domestic dog & & & 6 & 4 & 0.54 & $3,528-4,096$ \\
\hline 家羊 Domestic goat/sheep & & & 1 & 1 & 0.09 & 3,637 \\
\hline 家牛 Domestic cow/yak & & & 842 & 43 & 76.46 & $3,490-4,219$ \\
\hline 家马 Domestic horse & & & 241 & 17 & 21.88 & $3,637-4,096$ \\
\hline
\end{tabular}

EN：濒危; VU: 易危; NT: 近危; LC: 无危

EN, Endangered; VU, Vulnerable; NT, Near Threatened; LC, Least Concern. 